\title{
Analysis on the Bacterial Resistance Mechanism and the Rational Use of Antibiotics

\author{
Jingai Wen ${ }^{1, a}$, Yan Dong ${ }^{2, *}$, Hong Cui ${ }^{3}$ \\ ${ }^{1}$ Community Health Centre, Hongqi Hospital, Mudanjiang Medical University, Aimin District, \\ Mudanjiang, 157011, China;
}

${ }^{2}$ Pathogenic biology and Immunology Laboratory, Mudanjiang Medical University, Mudanjiang, 157011, China;

${ }^{3}$ Department of Pharmacy, Hongqi Hospital, Mudanjiang Medical University, Aimin District, Mudanjiang, 157011, China;

awenwen@126.com

*Corresponding author yandong@163.com

Keywords: Bacteria; Antibiotics; Reasonable use; Role

\begin{abstract}
The Bacteria is as an ancient creature that appears on the earth long before human beings, it is the world's most widely distributed organisms; humans' infection in parts of the bacteria causes the human body with inflammation, the clinical mainly uses the antibiotics as antibacterial drugs. In recent years, because of the abuse of the antibiotics and the bacterial resistance in order to adapt to the environment evolution, some bacteria also have a higher toxicity. The drug resistance of bacteria becomes the thorny issue of clinical treatment in recent years, and is paid more and more attention. This article is mainly through the analysis of the mechanism of drug resistance of bacteria and antibiotic action principle, and how to explore the clinical use of antibiotics in response to the drug resistance of bacteria.
\end{abstract}

\section{Introduction}

The antibiotics used antibiotics, such as bacteria, fungi, actinomycetes microbe in the process of the life activities of metabolites, for a variety of pathogenic microorganisms have strong inhibition and killing effect, in very low concentrations can selectively kill his species or inhibit its function. Antibiotic mainly adopts the methods for production of microbial fermentation, such as tetracycline, penicillin, etc; there are a few antibiotics such as thiamphenicol toxin, fluorine benzene nicol, chloramphenicol available chemical methods such as synthesis of. In addition, transformed by the molecular structure of natural antibiotics or taking microbial fermentation product as precursors to produce a large amount of semisynthetic antibiotics, such as cephalosporin, ampicillin, etc. Widely used in recent years, antibiotics, but because of the abuse of antibiotics and bacteria to adapt to the environment caused by factors such as their own evolution led to the production of bacterial drug resistance, it brings many problems for clinical anti-infection treatment. In this paper, the paper discusses mechanism of bacterial drug resistance; provide reference for clinical rational use of antibiotics.

\section{The bacterial resistance mechanisms}

\section{The classification of the bacterial drug resistance}

The drug-resistant bacteria are divided into two kinds, genetic resistance and external resistance. Genetic resistance is refers to the bacteria through its own evolution and mutation, make it no longer sensitive to antibiotics, and resistance. There are divided into two kinds: one kind is congenital, resistant bacteria innate immune to certain antibiotics, is determined by its chromosome genes, such as streptococcus of aminoglycoside antibiotics, pseudomonas aeruginosa to most antibiotics, etc.; Another is produced by genetic, because natural antibiotics are produced by the 
bacteria to protect themselves against other bacteria, human improvement of these antibiotics to control some bacteria, the bacteria through the long-term evolution, by changing their chromosome structure, gradually acquired immune to these antibiotics, drug resistance was the result. Acquired resistance is due to bacteria, in contact with antibiotics against by changing its own characteristics, make the effect of antibiotics on the culling weakened or even failure, and avoid being culling. Acquired drug resistance may be because of drug resistance to other antibiotics kill harmful bacteria, the survivors were offered the opportunity to genetic resistance gene, bacteria by changing the chromosomes from generation to generation, and become the inherent drug-resistant bacteria, may also be lost due to no longer use some sort of antibiotic resistance.

\section{The mechanism of the bacterial drug resistance}

Avenues for drug-resistant bacteria mainly through the following aspects: 1) the bacteria by producing passivation enzyme acquired drug resistance. After contact with the antibiotics, the bacteria are by producing some hydrolysis or change the antibiotic activity of the enzyme against antibiotics, and with the resistance. The main enzyme bacteria are beta, amino sugar phthalein amine enzyme in two classes' inactivated enzyme, acetoxy transferase, DNA gyrase, etc. Beta phthalein amine enzymatic antibiotics can be damaged in the beta phthalein amine ring, disable beta phthalein amine within a class of antibiotics, such as broad-spectrum cephalosporin, and ammonia broad-spectrum penicillin resistance was born. Amino sugars by acting on two kinds of inactivated enzyme amino sugar - NH on two classes of antibiotics related carbon atoms, or groups - $\mathrm{OH}$, reduces the chance of antibiotics combined with bacterial ribosome, and lose the antibiotic effect. The mechanism of action of several other enzymes is similar to the above two enzymes based wood, just according to different antibiotics. 2) The bacteria by changing the targeted protein to obtain the resistance. Bacteria, there are many can be combined with antibacterial drugs targeted, bacteria in the role of antibiotics and, by changing their targeted, the combination of antibiotics and gumming mutates, less sensitive to make the bacteria to drugs, to protect yourself, resistance, such as methicillin-resistant staphylococcus aureus. 3) The bacteria by changing cell membrane permeability and the membrane pump discharge for drug resistance. Bacteria through the cell membranes to reduce the through capacity of antimicrobial drugs, at the same time, the bacteria to reduce the concentration of antibiotics in the cell and the initiative will enter inside the membrane exudes, drugs to reduce the effect of antibiotics on bacteria and drug resistance. Bacterial cell membranes of protection in such as gram negative bacilli to penicillin, pseudomonas aeruginosa and other gram-negative bacilli to some classes and broad-spectrum penicillin, including third-generation cephalosporin, cephalosporin class of antibiotics, bacterial resistance to tetracycline was mainly produce new protein block cell walls make drugs cannot enter, gram-negative bacilli to amino sugar jam principle of two classes are similar. Outside mechanism mainly exist negative bacillus of wow ketones and the big ring vinegar class antibiotic resistance, staphylococcus aureus resistant to tetracycline, etc.

\section{The principle of the reasonable use of antibiotics}

Due to other reasons such as incorrect use of antibiotics, the drug-resistant bacteria appear constantly, make the mankind is faced with serious challenges, and reasonable use of antibiotics becomes the key to reduce the bacterial drug resistance. To do reasonable use of antibiotics, must master the commonly used antibiotic mechanism, pharmacokinetics, adverse reactions and bacteria resistant mechanism.

First, grasp the pharmacological effects of the antibiotics; suit the remedy to the case. Second, we should according to disease condition, to determine the antibiotic medicine method, the dosage and course of treatment. Third, we should pay attention to bacterial drug resistance. Be careful not to low dose of an antibiotic for a long time, to use drugs, according to the situation in turn not to abuse of broad-spectrum antibiotics. Fourth, pay attention to when using the interaction of various antibiotics at the same time, should also pay attention to the antibiotics don't arbitrary mixed with other drugs. Fifth, pay attention to all kinds of drug withdrawal period and doping period. 
To strengthen the rational use of antibiotics, can effectively control the harm of patients with some antibiotics, because of the adverse reactions of antibiotics because of individual different and different, so ask for every patient carefully and test whether have allergic reactions to certain antibiotics or some kind of antibiotics, only ensures the rational use of antibiotics, would let patients can effect in the process of use of antibiotics.

\section{The unreasonable situation of the clinical antibiotic using}

Antibiotics not only part of bacteria, actinomyces, fungi, helix, such as chlamydia and rickettsia role, and some antibiotics and antiviral, resisting parasites and promote animal growth. The kinds of antibiotics are more, mainly used in the aquaculture of tetracycline drugs, aminoglycoside drugs and amide alcohols, etc. Or according to the scope of the antibiotics antibacterial function etc, can be divided into the following categories: (1) main function positive of bacteria antibiotics, such as penicillin and cephalosporin, forests amine etc. (2) the main effect the negative bacteria antibiotics, such as streptomycin, gentamicin, kanamycin and neomycin and much slime molds, etc.; (3) broad spectrum antibiotics, such as tetracycline drugs and chloramphenicol drugs, not only for gram positive bacteria, negative effect, for some mycoplasma, chlamydia, helix is effective; (4) antifungal drugs, such as griseofulvin, nystatin and amphotericin, etc. (5) parasite drug resistance, such as salinomycin, ivermectin, the toxin A and mo can rhzomorph, etc.; 6 forage antibiotics, such as Virginia, the bacitracin zinc, etc, have promote animal growth, improve the effect of production performance.

Penicillin and probenecid compatibility can make the present synergy penicillin blood drug concentration; and amoxicillin and enzyme production of drug resistance bacteria infection to amoxicillin has strong antibacterial action; cooperate with streptomycin, gentamicin to treat serious mixed infection. The hloramphenicol, erythromycin, tetracycline, large ring lactone class have some interference from the bactericidal activity to penicillin, should not be used. Gentamicin is unfavorable and compatibility with penicillin intravenous drip, two drug compatibility must be separately for medicine; Compound new Ming bacteriostatic agent for slow effect, penicillin is breeding season fungicide, two drug compatibility effect of penicillin bactericidal action; Heavy metal ions (copper, zinc, mercury, etc.), acid, iodine, oxidant, reductant, hydroxyl compounds can damage the activity of the penicillin, taboo compatibility. Penicillin to animal toxicity was low, but easy to cause allergic animals. When large doses of penicillin, but nerve symptoms, such as coma, convulsions, hyperreflexia, etc.

Compatibility of the Terramycin and tetracycline drugs treatment of gastrointestinal and respiratory infections often synergy; with sodium sulfate by 1:1 proportion at the same time, is conducive to the absorption of Terramycin. Terramycin share have antagonism with penicillin; Taken with zinc sulfate, B vitamins, while can reduce drug efficacy; Containing divalent ions such as calcium, aluminum, magnesium, iron, etc. In the full price of feed, can form complex, thus reducing effect; With cephalosporin, sulfa drugs and novobiocin joint use, can be toxic and side effects on the liver; Can't and josamycin, doxycycline share in the north. Large ring lactone class and lincomycin drugs and share possible antagonism effect, thus unfavorable combination; and $\mathrm{p}$ a lactam medicines, due to the product of rapid bacteriostasis, interact can. Fluorine benzene test for thiamphenicol former single fluorine derivatives, antibacterial spectrum and antibacterial activity was slightly better than the chloramphenicol and thiamphenicol toxin. $70 \mathrm{~s}$ ribosomal subunit, inhibit bacteria and $50 \mathrm{~s}$ subunit, inhibiting peptide acyltransferase activity, also an extension of the inhibitory peptides, which interfere with the bacterial protein synthesis, for a variety of gram-positive bacteria and gram-negative bacteria and mycoplasma has strong antibacterial activity. Inhibits the red blood cells, white cells and platelets, leukopenia phenomenon may occur after taking this medicine. 


\section{The countermeasures of the clinical rational using of the antibiotics}

Antibiotics not only part of bacteria, actinomyces, fungi, helix, such as chlamydia and rickettsia role, and some antibiotics and antiviral, resisting parasites and promote animal growth.

The kinds of antibiotics are more, mainly used in the aquaculture of tetracycline drugs, aminoglycoside drugs and amide alcohols, etc. Or according to the scope of the antibiotics antibacterial function etc, can be divided into the following categories: (1) the main function positive of bacteria antibiotics, such as penicillin and cephalosporin, forests amine etc. (2) the main effect negative bacteria antibiotics, such as streptomycin, gentamicin, kanamycin and neomycin and much slime molds, etc.; (3) broad spectrum antibiotics, such as tetracycline drugs and chloramphenicol drugs, not only for gram positive bacteria, negative effect, for some mycoplasma, chlamydia, helix is effective; (4) antifungal drugs, such as griseofulvin, nystatin and amphotericin, etc. (5) parasite drug resistance, such as salinomycin, ivermectin, the toxin A and mo can rhzomorph, etc.; 6 forage antibiotics, such as Virginia, bacitracin zinc, etc, have promote animal growth, improve the effect of production performance.

Clinical doctors generally believe that new drug is better than older drugs, actually should be particular case is particular analysis. If the cephalosporin's in the process of development, there is a first, second and third generation. The third generation cephalosporin and than the first generation has many advantages, such as negative bacilli resistant effect is stronger, the beta lactamase stability. Effective for pseudomonas aeruginosa, wide antimicrobial spectrum, the anaerobic bacteria also have certain curative effect. But there are not as good as the first generation cephalosporins that affect on staphylococcus aureus, for example, the first generation cephalosporin, so for severe infections should be chosen first generation instead of the third generation. Another problem in selecting varieties of antibiotics is a little note similar varieties on the role of difference. Think similar species are similar, actually otherwise. Role not only strength is different; sometimes role characteristics also have very big difference. Such as gentamicin and kanamycin, aminoglycoside antibiotics, have broad spectrum antimicrobial effects, but the antibacterial spectrum, antibacterial activity, the characteristics of clinical pharmacology, indications and therapeutic dose have some difference.

Any therapeutic drugs have duality; its healing one side, and toxic side effects. Antimicrobial agents are no exception. Penicillin, for example, can cause anaphylactic shock, streptomycin can cause deafness, chloramphenicol can induce aplastic anemia, etc. Long-term application of antimicrobial agents can make the bacteria resistant strains, and even cause double infection. All of these show antibacterial drugs have toxic effects on the body. In the above phenomenon is not completely is not the result of the rational use of antimicrobial agents. Therefore, emphasizing the rational use of antimicrobial agents is very important. Rational use of antimicrobial agents, including in the diagnosis of clear premise (clear pathogenic microorganism), choose one or two kinds of antimicrobial agents, adopt appropriate dosage and course of treatment, to achieve the purpose of eliminating infectious microorganisms. At the same time to enhance the body's resistance during treatment the force and immunity. So that the antimicrobial agents to achieve twice the result with half the effort. Rational use of antimicrobial agents, should determine the pathogenic microorganisms of infectious diseases, blood, urine, sputum culture should be done before using this drug. Conditional place will do even anaerobic bacteria culture and drug sensitivity test.

Anti-infective drug combination rate as high as $68.74 \%$. As domestic hospitals have to cefazolin, gentamicin, erythromycin and penicillin with use cases. And vitamin C, metronidazole, cefoperazone, tobramycin, and compound new Ming, ampicillin, prednisone and ketone health with use cases, 8 kinds of drug 5 classes of antimicrobials used simultaneously or successively, have effect on $G$ + cocci ampicillin, acting on the $G$ - and $G$ + cocci cefoperazone, effect on pseudomonas aeruginosa tobramycin, bacteriostatic action of compound new Ming, and ACTS on the anaerobic bacteria metronidazole, will eventually lead to dysbacteriosis, fungal infections, and had to use a ketone health. Antibacterial drug combination in use there are a lot of problems. According to the principle of sterilization and bacteriostasis, generally divided into three categories: 
(1) organic fungicide: can quickly kill bacteria breeding season, such as penicillin class (including) semi-synthetic penicillin, cephalosporin, vancomycin, bacitracin, etc. (2) a slow disinfectant: kill bacteria stationary phase, such as more slime molds, aminoglycoside, rifamycin class, etc. (3) bacteriostatic agent available: mainly inhibit the growth of bacteria, such as tetracycline, chloramphenicol, large ring lactone class, clindamycin, etc. The first (1) and (2) class combination is commonly synergy, and the first (1) and (3) class combination, is more like the antagonism. But it must be emphasized that many cases and concrete analysis. For example, there are a number of cases to penicillin and ampicillin, both for the medicine, is a major role in $\mathrm{G}+$ cocci, combination can only increase adverse reactions and cost, to increase curative effect. As another example, cephalosporin class and aminoglycoside antibiotics, the former is the beta lactam amino sugars connected with the latter, the activity of amino sugar reduced or failure, obviously combination is not reasonable.

To speed up the research direction of new drug research and development at present mainly concentrated in the following aspects: the development of new drugs, high stability, and enzyme inhibitor, such as the development of phthalein amine enzyme in beta is not sensitive to antibiotics or inhibits beta phthalein amine enzymatic antibiotics; Improve the level of repressor protein, and regulate the expression of efflux gene against outside system caused by bacteria resistant. However, just relying on the research on new drugs, and can prolong the service life of antibiotics, only in the medical units to establish the management system of rational use of antibiotics, is the most effective method.

\section{Conclusion}

The standard used in the antibiotics method is according to the bacterial culture and drug sensitive test, the application of bacteria killing one of the most effective antibiotics. The bacterial resistance mechanisms and the biochemical interactions between bacteria and antibiotics are very complex; the germs multiply in the constantly evolving at the same time. The new resistance mechanisms were found, humans and the bacteria war would be long and hard, this is essential in the process of bacteria and human evolution. The bacteria resistant bacteria is the root cause of genetic variation, and the application of antibiotics has induced the role of genetic variation, so the reasonable use of the antibiotics needs to cause the attention of all the medical staff.

\section{Acknowledgement}

This research was financially supported by the National Science Foundation.

\section{References}

[1] Mayadah Shehadeh, Ghadeer Suaifan, Rula M. Darwish, Mayyada Wazaify, Luna Zaru, Suzan Alja'fari. Knowledge, attitudes and behavior regarding antibiotics use and misuse among adults in the community of Jordan. A pilot study[J]. Saudi Pharmaceutical Journal. 2011 (2)

[2] Lucy Reynolds, Martin McKee. Factors influencing antibiotic prescribing in China: An exploratory analysis[J]. Health policy. 2012 (1)

[3] C. Kristiansson, M. Reilly, E. Gotuzzo,H. Rodriguez, A. Bartoloni, A. Thorson,T. Falkenberg, F. Bartalesi,G.Tomson,M.Larsson. Antibiotic use and health - seeking behaviour in an underprivileged area of Perú[J]. Tropical Medicine \& International Health. 2012 (3)

[4] P. M.Hawkey. Prevalence and clonality of extended - spectrum $\beta$ - lactamases in Asia[J]. Clinical Microbiology and Infection. 2012(10)

[5] Naveen Shrestha, K. C. Samir, Rob Baltussen, Kumud K.Kafle,David Bishai, Louis Niessen. Practical Approach to Lung Health in Nepal: better prescribing and reduction of cost[J]. Tropical Medicine \& International Health . 2013(5) 
[6] R. Colodner, W. Rock, B. Chazan,N. Keller, N. Guy,W. Sakran, R. Raz. Risk Factors for the Development of Extended-Spectrum Beta-Lactamase-Producing Bacteria in Nonhospitalized Patients[J]. European Journal of Clinical Microbiology \& Infectious Diseases . 2012 (3)

[7] Margaret J. Emslie, Christine M. Bond. Public knowledge, attitudes and behaviour regarding antibiotics[J]. European Journal of General Practice . 2003 (3)

[8] Nguyen T.K Chuc, Mattias Larsson, Nguyen T Do, Vinod K Diwan, Goran B Tomson, Torkel Falkenberg. Improving private pharmacy practice[J]. Journal of Clinical Epidemiology. 2012 (11) [9] Mark A. Schuster, Elizabeth A. McGlynn, Robert H. Brook. How Good Is the Quality of Health Care in the United States?[J]. Milbank Quarterly. 2012 (4)

[10] Connell SR, Tracz DM, Nierhaus KHet al. Ribosomal protection proteins and their mechanism of tetracycline resistance. Antimicrobial Agents and Chemotherapy. 2012(10)

[11] Morse S A,Johnson S R,Riddle J W, et al.High-level tetracycline resistance in Neisseriagonorrhoeae is result of acquisition of Streptococcal tetM determinant. Antimicrobial Agents and Chemotherapy. 2011(08)

[12] Hansen L M, Blanchard P C, Hirsh D C. Distribution of tet (H)among Pasteurella isolates from the United States and Canada. Antimicrobial Agents and Chemotherapy. 2012(06) 